\title{
The Principle of Limit Amplitude for Symmetric Hyperbolic Systems of First Order in the Half-Space $\mathbf{R}_{+}^{\boldsymbol{n}}$
}

\author{
By \\ Seiichiro WAKABAYASHI*
}

\section{§1. Introduction}

The present paper is concerned with the principle of limit amplitude for symmetric hyperbolic systems in a half-space. Our proof for the validity of the principle is based on the eigenfunction expansion theorem established in the preceding paper [3]. For the notation and terminology in this paper we refer the reader to [3]. We shall consider the following mixed initial-boundary value problem for hyperbolic systems:

$$
\frac{\partial}{\partial t} u(t, x)=i A u(t, x)+\frac{1}{i} e^{i k t} f(x), \quad t>0, \quad x \in \mathbb{R}_{+}^{n},
$$

$$
\left.B u(t, x)\right|_{x_{n}=0}=0
$$

$$
u(0, x)=g(x),
$$

where $k(\neq 0)$ is a real number,

$$
A=\frac{1}{i} \sum_{j=1}^{n} A_{j} \frac{\partial}{\partial x_{j}}
$$

the $A_{j}$ are $2 m \times 2 m$ constant Hermitian matrices, $B$ is an $m \times 2 m$ constant matrix with rank $m$ and $u(t, x), g(x)$ and $f(x)$ are vector-valued functions whose values lie in $\mathbf{C}^{2 m}$. Replacing $u(t, x)$ in (1.1) and (1.2) by $e^{i k t} v(x)$, we obtain the corresponding stationary problem:

$$
(A-k I) v(x)=f(x), \quad x \in \mathbf{R}_{+}^{n},
$$

Communicated by S. Matsuura, September 9, 1974.

* Department of Mathematics, Tokyo University of Education, Tokyo. 


$$
\left.B v(x)\right|_{x_{n}=0}=0
$$

We assume the following conditions:

(L.1) $L \equiv\left(I \frac{\partial}{\partial t}-i A\right)$ is uniformly propagative.

(L.2) The operator $A$ is elliptic.

(L.3) The multiplicity of the real roots of $\left.Q(\lambda, \eta)\right|_{\eta=(\xi, \tau)}=0$ with respect to $\tau$ is not greater than two for every $\xi \in \Xi^{n-1}$ and real $\lambda \neq 0$. Moreover the equation has at most only one couple of real double roots for every $(\xi, \lambda) \neq(0,0)$.

(B.1) The boundary matrix $B$ is minimally conservative.

Under the above assumptions the following expansion theorem was proved in [3]:

Theorem 1 (cf. Theorem 6.2 in [3]). Assume that the conditions (L.1)-(L.3) and (B.1) are satisfied and that $f \in L^{2}\left(\mathbf{R}_{+}^{n}\right)$.

(i) The expansion formula

$$
\begin{aligned}
P f(x)= & \left.\sum_{j=1}^{2 \rho} \int_{\Xi^{n}} \Psi\right)_{j}^{ \pm}(x, \eta) \hat{f}_{j}^{ \pm}(\eta) d \eta \\
& +\sum_{v=1}^{s} \sum_{j=1}^{2 \rho} \int_{D_{v} \times \Xi} \Psi \frac{j}{j}+2 v \rho(x, \eta) \hat{f}_{j+2 v \rho}^{ \pm}(\eta) d \eta
\end{aligned}
$$

holds, where

$$
\hat{f}_{j+2 v \rho}^{ \pm}(\eta)=\int_{\mathbf{R}_{+}^{n}} \Psi_{j+2 v \rho}^{ \pm}(x, \eta)^{*} f(x) d x, \quad 1 \leqq j \leqq 2 \rho, 1 \leqq v \leqq s .
$$

Here the above integrals are taken in the sense of the limit in the mean and $P$ is the orthogonal projection onto $R(\mathbf{A})^{a}=N(\mathbf{A})^{\perp}$.

(ii) $f \in D(\mathbf{A})$ if and only if $\lambda_{j}(\eta) \hat{f}_{j}^{ \pm}(\eta) \in P_{j}(\eta) L^{2}\left(\Xi^{n}\right), k_{v}(\xi) \hat{f}_{j+2 v \rho}^{ \pm}$ $\in P_{j}(\eta) L^{2}\left(D_{v} \times \Xi\right), 1 \leqq j \leqq 2 \rho, 1 \leqq v \leqq s . \quad$ Then

$$
\begin{aligned}
(\mathbf{A} f)(x)= & \sum_{j=1}^{2 \rho} \int_{\Xi n} \lambda_{j}(\eta) \Psi_{j}^{ \pm}(x, \eta) \hat{f}_{j}^{ \pm}(\eta) d \eta \\
& +\sum_{v=1}^{s} \sum_{j=1}^{2 \rho} \int_{D_{v} \times \Xi} k_{v}(\xi) \Psi_{j+2 v}^{ \pm}(x, \eta) \hat{f}_{j+2 v \rho}^{ \pm}(\eta) d \eta,
\end{aligned}
$$




$$
(\mathbf{A} f) \hat{\boldsymbol{j}}^{ \pm}(\eta)=\lambda_{j}(\eta) \hat{f}_{\hat{j}}^{ \pm}(\eta), \quad 1 \leqq j \leqq 2 \rho,
$$

$$
(\mathbf{A} f)_{\hat{j}^{+}+2 v \rho}(\eta)=k_{v}(\xi) \hat{f}_{\dot{j}+2 v \rho}^{+}(\eta), \quad 1 \leqq j \leqq 2 \rho, \quad 1 \leqq v \leqq s
$$

Here the $\lambda_{j}(\eta)$ are distinct roots of $Q(\lambda, \eta)=0$ and the $k_{v}(\xi)$ are nonvanishing zeros of the Lopatinski determinant (see [3]).

Remark. The differential operator $A$ defines an unbounded linear operator $\mathscr{A}$ in $L^{2}\left(\mathbf{R}_{+}^{n}\right)$ with domain

$$
D(\mathscr{A})=\left\{v(x) \in C_{0}^{\infty}\left(\overline{\mathbf{R}_{+}^{n}}\right) ;\left.B v(x)\right|_{x_{n}=0}=0\right\} .
$$

Thus we denoted by $\mathbf{A}$ the closure of $\mathscr{A}$ in [3], which is a self-adjoint operator in $L^{2}\left(\mathbf{R}_{+}^{n}\right)$.

In [3] it was proved that $\sigma(\mathbf{A})=\mathbf{R}^{1}$. Hence in general there exist no solutions of (1.5) and (1.6) belonging to $L^{2}\left(\mathbb{R}_{+}^{n}\right)$ for real $k$ and $f \in$ $L^{2}\left(\mathbf{R}_{+}^{n}\right)$. Then the question arises of determining uniquely a solution of (1.5) and (1.6). There are three important approaches of deriving a unique solution of (1.5) and (1.6), i.e., the radiation principle, the limiting absorption principle and the limiting amplitude principle. The limiting absorption principle for (1.5) and (1.6) was justified in $M$. Matsumura [2] under more restrictive assumptions than ours.

In this paper the following theorems will be proved:

Theorem 2. Let $f(x)=0$ in (1.1), $g(x) \in D(\mathbf{A})$ in (1.3) and Supp $g(x)$ be bounded. Suppose that at least one of the following conditions holds: a) $E^{+}(\xi ; 0) \cap \mathscr{B}=\{0\}$ for $|\xi| \neq 0$, b) $g(x)$ belongs to $C^{\infty}\left(\overline{\mathbf{R}_{+}^{n}}\right)$. Then for the solution $u(t, x)$ of the equations (1.1)-(1.3) and any compact set $K \subset \overline{\mathbf{R}_{+}^{n}}$

$$
\frac{d^{l}}{d t^{l}} u(t, x) \longrightarrow \delta_{0, l} g_{2}(x) \text { in } L^{2}(K) \text { as } t \longrightarrow \infty, l=0,1
$$

hold, where $\delta_{0, l}=1$ for $l=0,=0$ for $l=1$, and $g=g_{1}+g_{2}, g_{1} \in N(\mathbf{A})^{\perp}$ $=R(\mathbf{A})^{a}, g_{2} \in N(\mathbf{A})$. Moreover if a) holds, then $g_{2}=0$.

Theorem 3 (the limiting amplitude principle). Let $f \in L^{2}\left(\mathbf{R}_{+}^{n}\right), g$ $\in D(\mathbb{A})$ and $k \neq 0$ be real in (1.1)-(1.3) and $\operatorname{Supp} f(x)$ and $\operatorname{Supp} g(x)$ 
be bounded. Suppose that at least one of the following conditions holds: a) $E^{+}(\xi ; 0) \cap \mathscr{B}=\{0\}$ for $|\xi| \neq 0$, b) $f(x)$ and $g(x)$ belong to $C^{\infty}\left(\overline{\mathbf{R}_{+}^{n}}\right)$. Then for the solution $u(t, x)$ of the equations (1.1)-(1.3) and any compact set $K \subset \overline{\mathbf{R}_{+}^{n}}$

$$
\begin{aligned}
& e^{-i k t}\left[\frac{d^{l}}{d t^{l}} u(t, x)-\delta_{0, l}\left(\frac{1}{k} f_{2}+g_{2}\right)\right] \longrightarrow(i k)^{l} v(x) \text { in } L^{2}(K) \\
& \text { as } t \longrightarrow \infty, \quad l=0,1
\end{aligned}
$$

hold, where $v(x) \in L_{\text {loc }}^{2}\left(\overline{\mathbf{R}_{+}^{n}}\right)^{1)}$ and $f=f_{1}+f_{2}, f_{1} \in N(\mathbf{A})^{\perp}, f_{2} \in N(\mathbf{A})$. Moreover $\alpha_{R}(x) v(x) \in D(\mathbf{A})$ and $v(x)$ satisfies (1.5) in the sense of distribution and also satisfies (1.5) and (1.6) in the following sense: There exists $a$ sequence $\left\{v_{j}\right\} \subset H^{1}\left(\mathbf{R}_{+}^{n}\right)$ such that $\left.B v_{j}\right|_{x_{n}=0}=0, v_{j} \rightarrow v(x)$ in $L_{\text {loc }}^{2}\left(\overline{\mathbf{R}_{+}^{n}}\right)$ and $(A-k I) v_{j} \rightarrow f$ in $L_{\text {loc }}^{2}\left(\overline{\mathbf{R}_{+}^{n}}\right)$ as $j \rightarrow \infty$. Here $\alpha_{R}(x)=1$ for $|x| \leqq R$, $=0$ for $|x| \geqq R+1$ and belongs to $C^{\infty}\left(\mathbf{R}_{+}^{n}\right)$.

Remark 1. a) $E^{+}(\xi ; 0) \cap \mathscr{B}=\{0\}$ for $|\xi| \neq 0$ implies the coercivity of $B$ for the elliptic operator $A$. Thus if a) holds, then

$$
(1.14)^{\prime} e^{-i k t} \frac{d^{l}}{d t^{l}} u(t, x) \longrightarrow(i k)^{l} v(x) \quad \text { in } H_{l o c}^{1}\left(\overline{\mathbf{R}_{+}^{n}}\right) \quad \text { as } \quad t \longrightarrow \infty, l=0,1
$$

and $v(x)$ is a solution of (1.5) and (1.6) belonging to $H_{1 \text { oc }}^{1}\left(\overline{\mathbf{R}_{+}^{n}}\right) .{ }^{2)}$

Remark 2. From Lemma 3 it is easy to see that

$$
(\mathbf{A}-\lambda \mathbf{I})^{-1} f(x) \longrightarrow v(x) \quad \text { in } \quad L_{\text {loc }}^{2}\left(\overline{\mathbf{R}_{+}^{n}}\right) \text { as } \lambda \longrightarrow k-i 0 .
$$

In order to prove the above theorems we state some lemmas and propositions in $\S 2$, following D. M. Eidus [1]. In $\S 3$ we shall give their proofs.

\section{§2. Preliminaries}

Let $\mathscr{H}$ be a separable Hilbert space, $\mathscr{H}_{0}$ a subspace of $\mathscr{H}, \widetilde{P}$ the orthogonal projection onto $\mathscr{H}_{0}$, and $A$ a self-adjoint operator in $\mathscr{H}$.

1) $L_{\text {loc }}^{2}\left(\overline{\mathbf{R}_{+}^{n}}\right)$ denotes the space of vector-valued functions $f$ such that $f \in L^{2}(K)$ for any compact set $K \subset \overline{\mathbf{R}_{+}^{n}}$.

2) $H_{\text {loc }}^{1}\left(\widehat{\mathbf{R}_{+}^{n}}\right)$ denotes the space of vector-valued functions $f$ such that $\alpha_{R}(x) \frac{\partial^{\alpha}}{\partial x^{\alpha}} f(x)$ $\left.\in L^{2} \overline{\left(\mathbf{R}_{+}^{n}\right.}\right)$ for $|\alpha| \leqq 1$ and any $R>0$. 
Definition 1. An element $f \in \mathscr{H}$ is said to satisfy the condition $T_{1}$ if for all real $a$ and $b(a \cdot b>0, a<b)$ there exists a constant $C$ such that for any complex $\lambda=k+\varepsilon i(\varepsilon \neq 0, k \in[a, b])$ the inequality

$$
\|\widetilde{P} R(\lambda) f\| \leqq C
$$

holds.

Lemma 1. Suppose that $f$ satisfies $T_{1}$. Then for almost all $k \in \mathbf{R}^{1}$ there exist the weak limits ${ }^{3}$

$$
\underset{\lambda \rightarrow k \pm i 0}{w-\lim _{\lambda \rightarrow 0}} \tilde{P} R(\lambda) f=v^{( \pm)}(k)
$$

This lemma can be proved by Fatou's theorem and Riemann's mapping theorem.

Lemma 2. Let $f$ satisfy $T_{1}$. Suppose that $F(k)$ is a complex-valued function, continuous and bounded on $(-\infty, 0) \cup(0, \infty)$. Then for any real $a$ and $b(a \cdot b>0, a<b$, including $a=-\infty$ and $b=\infty)$

$$
\widetilde{P} \int_{b}^{a} F(k) d E(k) f=\frac{1}{2 \pi i} \int_{b}^{a} F(k) \theta(k) d k
$$

holds, where $\{E(k)\}_{-\infty<k<\infty}$ denotes the right-continuous spectral family of $A$ and

$$
\theta(k)=v^{(+)}(k)-v^{(-)}(k) .
$$

Here the integral on the right-hand side of (2.3) is taken in the sense of Bochner's integral.

We first consider the Cauchy problem for $t>0$

$$
\begin{gathered}
\frac{d}{d t} u(t)=i A u(t)+\frac{1}{i} e^{i k t} f, \\
u(0)=g \in D(A) .
\end{gathered}
$$

The following proposition can be easily verified.

3) $\lambda \rightarrow k \pm i 0$ means that $\lambda \rightarrow k$ along any path that does not cross the real axis and is not tangent to it. 
Proposition 1. Let $f=0$ in (2.5), $g$ satisfy $T_{1}$ and $g \in D(A)$. Let $u(t)$ be the solution of (2.5), (2.6). Then

$$
\widetilde{P} \frac{d^{l} u}{d t^{l}} \longrightarrow \delta_{0, l} \tilde{P} g_{2} \quad \text { in } \mathscr{H} \quad \text { as } t \longrightarrow \infty, l=0,1 \text {, }
$$

hold, where $\delta_{0, l}=1$ for $l=0,=0$ for $l=1$, and $g=g_{1}+g_{2}, g_{1} \in N(A)^{\perp}$, $g_{2} \in N(A)$.

Definition 2. An element $f \in \mathscr{H}$ is said to satisfy the condition $T_{2}$ if it satisfies $T_{1}$ and if $\theta(k)$ determined by (2.4) satisfies a Hölder condition on any interval $[a, b] \subset \mathbf{R} \backslash\{0\}$.

Lemma 3. Let $f \in N(A)^{\perp}$ satisfy $T_{2}$. Then replacing the weak limits by the strong limits in (2.2) Lemma 1 holds. Moreover

$$
v^{( \pm)}(k)= \pm \frac{\theta(k)}{2}+\frac{1}{2 \pi i} \int_{-\infty}^{\infty} \frac{\theta\left(k_{1}\right)}{k_{1}-k} d k_{1}
$$

where the integral is taken in the sense of the principal value. $v^{( \pm)}(k)$ satisfy a Hölder condition on any interval $[a, b] \subset \mathbf{R} \backslash\{0\}$.

This lemma follows from Lemma 2.

Proposition 2. Let $f$ satisfy $T_{2}, g=0$ and $k \neq 0$ be real in (2.5), (2.6), and $u(t)$ be the solution of (2.5), (2.6). Then

$$
\begin{gathered}
e^{-i k t} \tilde{P}\left[\frac{d^{l}}{d t^{l}} u(t)-\delta_{0, l} \frac{1}{k} f_{2}\right] \longrightarrow(i k)^{l} v^{(-)}(k) \text { in } \mathscr{H} \\
\text { as } t \rightarrow \infty, \quad l=0,1,
\end{gathered}
$$

hold.

Using Lemmas 2 and 3, we can easily prove the above proposition.

Lemma 4. Let $f \in \mathscr{H}$ be such that for all $a$ and $b$ with $a \cdot b>0$, $a<b$, and any $h_{0} \in \mathscr{H}_{0}$ there exists $C_{0}>0$ such that for all complex $\lambda=k+\varepsilon i(k \in[a, b], \varepsilon \neq 0)$ the inequality

$$
\left|\left(R(\lambda) f, R(\bar{\lambda}) h_{0}\right)\right| \leqq C_{0}
$$


holds. Then $f$ satisfies $T_{2}$. Here $v^{( \pm)}(k)$ satisfy a Hölder condition with exponent 1 on an arbitrary interval $[a, b] \subset \mathbf{R} \backslash\{0\}$.

The above lemma follows from the equality

$$
\frac{d}{d \lambda}\left(R(\lambda) f, h_{0}\right)=\left(R(\lambda)^{2} f, h_{0}\right)=\left(R(\lambda) f, R(\bar{\lambda}) h_{0}\right) \text {. }
$$

\section{§3. Proof of the Theorems}

We first state the following

Lemma 5. Let $f(r)$ be a complex-valued function and $f(r) \in L^{1}(0$, $\infty) \cap C^{1+\alpha}(0, \infty),{ }^{4)} \alpha>0$. Then for all $a$ and $b(a \cdot b>0, a<b)$ there exists a constant $C_{0}(>0)$ such that for any $k \in[a, b]$

$$
\left|\int_{0}^{\infty} \frac{f(r)}{(r-\lambda)^{2}} d r\right| \leqq C_{0}
$$

holds, where $\lambda=k+i \varepsilon, \varepsilon \neq 0$.

Now let $f, h \in L^{2}\left(\mathbf{R}_{+}^{n}\right), \operatorname{Supp} f(x)$ and $\operatorname{Supp} h(x)$ be bounded and $\mathbf{A}$ the self-adjoint operator associated with (1.5) and (1.6). Then it follows from Theorem 1 that for $\operatorname{Im} \lambda \neq 0$

$$
\begin{gathered}
\left(R(\lambda)_{j}^{ \pm}(\eta)=\frac{1}{\lambda_{j}(\eta)-\lambda} \hat{f}_{j}^{ \pm}(\eta), \quad 1 \leqq j \leqq 2 \rho,\right. \\
(R(\lambda) f)_{j+2 v \rho}^{\dagger \pm}(\eta)=\frac{1}{k_{v}(\xi)-\lambda} \hat{f}_{j+2 v \rho}^{ \pm}(\eta), \quad 1 \leqq j \leqq 2 \rho, 1 \leqq v \leqq s,
\end{gathered}
$$

hold. We can replace $f$ by $h$ in (3.2) and (3.3). Moreover we have $P R(\lambda) f=R(\lambda) P f$. Thus by Theorem 5.10 in [3] we obtain

$$
\begin{aligned}
& (R(\lambda) P f, R(\bar{\lambda}) h)=\sum_{j=1}^{2 \rho} \int_{\Xi^{n}} \frac{1}{\left(\lambda_{j}(\eta-\lambda)^{2}\right.} \hat{f}_{\bar{j}}^{ \pm}(\eta) \cdot \overline{h_{j}^{ \pm}(\eta)} d \eta \\
& \quad+\sum_{j=1}^{2 \rho} \sum_{v=1}^{s} \int_{D_{v} \times \Xi} \frac{1}{\left(k_{v}(\xi)-\lambda\right)^{2}} \hat{f}_{j+2 v \rho}^{+}(\eta) \cdot \overline{\hat{h}_{j+2 v \rho}^{ \pm}(\eta)} d \eta .
\end{aligned}
$$

4) $C^{1+\alpha}(0, \infty)$ consists ${ }_{-}^{\text {mo }}$ of continuous functions whose derivatives satisfy a Hölder condition with exponent $\alpha$ on any bounded closed subinterval of $(0, \infty)$. 
Let us consider the case $1 \leqq j \leqq \rho .5) \quad$ Put $S_{j}=\left\{\eta \in \Xi^{n} ; \lambda_{j}(\eta)=1\right\}, r=\lambda_{j}(\eta)$ and $\omega=\frac{\eta}{\lambda_{j}(\eta)}, 1 \leqq j \leqq \rho$. Then

$$
\begin{aligned}
& \int_{\Xi^{n}} \frac{1}{\left(\lambda_{j}(\eta)-\lambda\right)^{2}} \hat{f}_{\bar{j}}^{ \pm}(\eta) \cdot \overline{\hat{h}_{j}^{ \pm}(\eta)} d \eta \\
& \quad=\int_{0}^{\infty} \frac{r^{n-1}}{(r-\lambda)^{2}} d r \int_{S_{j}}\left(\omega \cdot n_{j}(\omega)\right) \hat{f}_{\bar{j}}^{ \pm}(r \omega) \cdot \overline{h_{j}^{ \pm}}(r \omega) d S_{j}
\end{aligned}
$$

holds, where $n_{j}(\omega)$ denotes the outward unit normal to $S_{j}$ at $\omega$ and $d S_{j}$ the surface element on $S_{j}$. Put

$$
I_{j}(r)=\int_{S_{j}}\left(\omega \cdot n_{j}(\omega)\right) \hat{f}_{j}^{ \pm}(r \omega) \cdot \overline{h_{j}^{ \pm}}(r \omega) d S_{j} .
$$

Let us prove below that $I_{j}(r) \in C^{\infty}(0, \infty)$. By Theorem 6.1 in [3] we have a local representation

$$
\begin{aligned}
\Psi_{j}^{ \pm}(x, \eta) & \\
& =\left\{\begin{array}{l}
0 \\
\left(\text { when there exists } l_{j}, 1 \leqq l_{j} \leqq \rho,\right. \text { such that } \\
\left.\tau_{l_{j}}^{+}\left(\xi ; \lambda_{j}(\eta) \pm i 0\right)=\sigma\right), \\
(2 \pi)^{-\frac{n}{2}} e^{i x \cdot \eta} P_{j}(\eta)+(2 \pi)^{-\frac{n}{2}} \sum_{\mu=1}^{p} e^{i x^{\prime} \cdot \xi} e^{i \tau_{\mu}\left(\xi \xi \lambda_{j}(\eta) \pm i 0\right) x_{n}} \\
\times C_{j \mu}(\eta)+(2 \pi)^{-\frac{n}{2}} e^{i x^{\prime} \cdot \xi} \sum_{p(l)=l_{j}}\left[\frac{\frac{\partial \lambda_{j}(\eta)}{\partial \tau}}{\overline{\Delta\left(\xi ; \lambda_{j}(\eta) \mp i 0\right)}}\right. \\
\quad \times \operatorname{det}\left(B h_{1}^{+}\left(\xi ; \lambda_{j}(\eta) \mp i 0\right), \ldots, B q_{v}\left(x_{n}, \xi ; \lambda_{j}(\eta) \mp i 0\right), \ldots\right. \\
\left.\left.\ldots, B h_{m}^{+}\right) h_{l}^{+}\left(\xi ; \lambda_{j}(\eta) \mp i 0\right)^{*}\right]_{v=1, \ldots, 2 m \downarrow} \\
\left(\text { when there exists } l_{j}, 1 \leqq l_{j} \leqq \rho,\right. \text { such that } \\
\left.\tau_{l_{j}}^{+}\left(\xi ; \lambda_{j}(\eta) \mp i 0\right)=\sigma\right),
\end{array}\right.
\end{aligned}
$$

5) $\lambda_{1}(\eta)>\lambda_{2}(\eta)>\cdots>\lambda_{\rho}(\eta)>0>\lambda_{\rho+1}(\eta)>\cdots>\lambda_{2 \rho}(\eta)$ for $\eta \neq 0$. 
where the $C_{j \mu}(\eta)$ are $2 m \times 2 m$ matrices whose elements are positively homogeneous of degree 0 . Noting that the $h_{l}^{+}\left(\xi ; \lambda_{j}(\eta) \mp i 0\right), \frac{\partial \lambda_{j}(\eta)}{\partial \tau}$ and $\Delta\left(\xi ; \lambda_{j}(\eta) \mp i 0\right)$ are positively homogeneous of degree 0 and that the $\tau_{\mu}^{+}\left(\xi ; \lambda_{j}(\eta) \pm i 0\right)$ are positively homogeneous of degree 1 , we obtain

$$
\begin{aligned}
& \frac{\partial^{k} \Psi \frac{ \pm}{j}(x, \eta)}{\partial r^{k}} \\
& =\left(\begin{array}{l}
0 \quad\left(\tau_{l_{j}}^{+}\left(\xi ; \lambda_{j}(\eta) \pm i 0=\sigma\right),\right. \\
(2 \pi)^{-\frac{n}{2}}\left(\frac{i x \cdot \eta}{r}\right)^{k} e^{i x \cdot \eta} P_{j}(\eta)+(2 \pi)^{-\frac{n}{2}} \sum_{\mu=1}^{p}
\end{array}\right. \\
& \left(\frac{i x^{\prime} \cdot \xi+i \tau_{\mu}^{+}\left(\xi ; \lambda_{j}(\eta) \pm i 0\right) x_{n}}{r}\right)^{k} e^{i x^{\prime} \cdot \xi} e^{i \tau_{\mu}^{+}\left(\xi ; \lambda_{J}(\eta) \pm i 0\right) x_{n}} C_{j \mu}(\eta) \\
& +(2 \pi)^{-\frac{n}{2}} e^{i x^{\prime} \xi} \sum_{p(l)=l_{j}}\left[\frac{\frac{\partial \lambda_{j}(\eta)}{\partial \tau}}{\Delta\left(\xi ; \lambda_{j}(\eta) \mp i 0\right)} \sum_{h=0}^{k}\left(\begin{array}{l}
k \\
h
\end{array}\right)\left(\frac{i x^{\prime} \cdot \xi}{r}\right)^{h}\right. \\
& \times \overline{\operatorname{det}\left(B h_{1}^{+}, \ldots, \frac{\partial^{k-h}}{\partial r^{k-h}} B q_{v}\left(x_{n}, \xi ; \lambda_{j}(\eta) \mp i 0\right), \ldots, B h_{m}^{+}\right)} \\
& \left.h_{l}^{+}\left(\xi ; \lambda_{j}(\eta) \mp i 0\right)^{*}\right]_{v=1, \ldots, 2 m \downarrow}\left(\tau_{l_{j}}^{+}\left(\xi ; \lambda_{j}(\eta) \mp i 0\right)=\sigma\right), \\
& k=1,2, \ldots
\end{aligned}
$$

Here we have used the relation $\frac{\partial}{\partial r}=\sum_{k=1}^{n} \frac{\eta_{k}}{r} \frac{\partial}{\partial \eta_{k}}$ and Euler's identity. It follows from the estimates of $\Psi_{j}^{ \pm}(x, \eta)$ in [3] that for fixed $a$ and $b$, $0<a<b$, and $r \in[a, b]$

$$
\left|\left(\frac{\partial^{k} \Psi \frac{ \pm}{j}}{\partial r^{k}}(x, \eta)\right) * f(x)\right| \leqq F_{j}^{k}(x, \omega)
$$

holds, where $F_{j}^{k}(x, \omega)$ satisfies the inequality

$$
\int_{S_{j}}\left(\omega \cdot n_{j}(\omega)\right)\left(\int_{\mathbf{R}_{+}^{n}}\left|F_{j}^{k}(x, \omega)\right| d x\right)^{2} d S_{j}<+\infty,
$$


since $\frac{|\eta|}{r} \leqq C, \operatorname{Supp} f(x)$ is bounded and $\frac{\partial^{i}}{\partial r^{i}} B q_{\nu}\left(x_{n}, \xi ; \lambda_{j}(\eta) \mp i 0\right)$ is continuous in $\left(x_{n}, \eta\right)$. Also we can replace $f(x)$ and $F_{j}^{k}(x, \omega)$ by $h(x)$ and $H_{j}^{k}(x, \omega)$ in (3.9) and (3.10), respectively. Therefore we have

$$
\begin{aligned}
& \frac{\partial^{k}}{\partial r^{k}} \hat{f}_{j}^{ \pm}(r \omega)=\int_{\mathbf{R}_{+}^{n}}\left(\frac{\partial^{k} \Psi_{j}^{+}}{\partial r^{k}}(x, \eta)\right)^{*} f(x) d x, \\
& \frac{\partial^{k}}{\partial r^{k}} \hat{h}_{j}^{ \pm}(r \omega)=\int_{\mathbf{R}_{+}^{n}}\left(\frac{\partial^{k} \Psi_{j}^{+}}{\partial r^{k}}(x, \eta)\right)^{*} h(x) d x .
\end{aligned}
$$

Thus we have

$$
\frac{\partial^{k}}{\partial r^{k}} I_{j}(r)=\int_{S_{j}}\left(\omega \cdot n_{j}(\omega)\right) \sum_{h=0}^{k}\left(\begin{array}{l}
k \\
h
\end{array}\right) \frac{\partial^{h}}{\partial r^{h}} \hat{f}_{j}^{ \pm}(r \omega) \cdot \overline{\frac{\partial^{k-h}}{\partial r^{k-h}} \hat{h}_{j}^{ \pm}(r \omega)} d S_{j}
$$

since

$$
\begin{aligned}
\left|\frac{\partial^{h}}{\partial r^{h}} \hat{f}_{j}^{ \pm}(r \omega) \cdot \overline{\frac{\partial^{k-h}}{\partial r^{k-h}} \hat{h}_{j}^{ \pm}(r \omega)}\right| \leqq & C\left\{\left(\int_{\mathbf{R}_{+}^{n}}\left|F_{j}^{h}(x, \omega)\right| d x\right)^{2}\right. \\
& \left.+\left(\int_{\mathbf{R}_{+}^{n}}\left|H_{j}^{k-h}(x, \omega)\right| d x\right)\right\}^{2}
\end{aligned}
$$

From this it follows that $I_{j}(r) \in C^{\infty}[a, b]$, i.e., $I_{j}(r) \in C^{\infty}(0, \infty)$. Put $\widetilde{S}_{v}=\left\{\eta \in \Xi^{n} ; k_{v}(\xi)=1, \xi \in D_{v}\right\}, D_{v}^{+}=\left\{\xi \in D_{v} ; k_{v}(\xi)>0\right\} \quad$ and $\quad D_{v}^{-}=D_{v} \mid D_{v}^{+}, 1$ $\leqq v \leqq s$. Moreover put $r=k_{v}(\xi)$ and $\omega=\frac{\eta}{k_{v}(\xi)}$ for $\xi \in D_{v}^{+}$. Then

$$
\begin{aligned}
& \int_{D_{v} \times \Xi} \frac{1}{\left(k_{v}(\xi)-\lambda\right)^{2}} \hat{f}_{j+2 v \rho}^{ \pm}(\eta) \cdot \overline{\hat{h}_{j+2 v \rho}^{ \pm}(\eta)} d \eta \\
& =\int_{D_{v}^{-} \times \Xi} \frac{1}{\left(k_{v}(\xi)-\lambda\right)^{2}} \hat{f}_{j+2 v \rho}^{ \pm}(\eta) \cdot \overline{\hat{h}_{j+2 v \rho}^{ \pm}(\eta)} d \eta \\
& \quad+\int_{0}^{\infty} \frac{r^{n-1}}{(r-\lambda)^{2}} d r \int_{\tilde{S}_{v}}\left|\omega \cdot \tilde{n}_{v}(\omega)\right| \hat{f}_{j+2 v \rho}^{ \pm}(r \omega) \cdot \overline{\hat{h}_{j+2 v \rho}^{ \pm}(r \omega)} d \tilde{S}_{v}
\end{aligned}
$$

holds, where $\tilde{n}_{v}(\omega)$ denotes the unit normal to $\tilde{S}_{v}$ at $\omega$ and $d \tilde{S}_{v}$ denotes the surface element on $\tilde{S}_{v}$. Put

$$
I_{j+2 v \rho}(r)=\int_{\tilde{S}_{v}}\left|\omega \cdot \tilde{n}_{v}(\omega)\right| \hat{f}_{j+2 v \rho}^{ \pm}(r \omega) \cdot \overline{\hat{h}_{j+2 v \rho}^{ \pm}(r \omega)} d \tilde{S}_{v} .
$$

By the definition of $\Psi_{j+2 v \rho}^{ \pm}(x, \eta)$ in [3] we see that 


$$
\begin{aligned}
& \Psi_{j+2 v \rho}^{ \pm}(x, \eta)=(2 \pi)^{-\frac{n}{2}} e^{i x^{\prime} \cdot \xi} \sum_{\mu=1}^{p} e^{i \tau_{\mu}^{+}\left(\xi ; k_{v}(\xi) \pm i 0\right) x_{n}} C_{j \mu}^{v}(\eta) \\
& +(2 \pi)^{-\frac{n}{2}} e^{i x^{\prime} \cdot \xi} \sum_{l=1}^{m}\left[\left(\frac{\lambda-k_{v}(\xi)}{\Delta(\xi ; \bar{\lambda})} \overline{\operatorname{det}\left(B h_{1}^{+}(\xi ; \bar{\lambda}), \ldots\right.}\right.\right. \\
& \left.\quad \ldots, B q_{\mu}\left(x_{n}, \xi ; \bar{\lambda}\right), \ldots, B h_{m}^{+}(\xi ; \bar{\lambda})\right)\left.\right|_{\lambda=k_{\nu}(\xi) \pm i 0} \\
& \left.\quad \times \gamma_{j l}^{v}(\eta)\right]_{\mu=1, \ldots, 2 m \downarrow}, \quad \eta \in D_{j^{\nu}},
\end{aligned}
$$

where the $C_{j \mu}^{v}(\eta)$ are $2 m \times 2 m$ matrices and positively homogeneous of degree 0 , and the $\gamma_{j l}^{v}(\eta)$ are $1 \times 2 m$ matrices and positively homogeneous of degree -1 . Thus we have

$$
\begin{aligned}
& \frac{\partial^{k} \Psi \frac{+}{j+2 v \rho}(x, \eta)}{\partial r^{k}}=(2 \pi)^{-\frac{n}{2}} \sum_{\mu=1}^{p}\left(\frac{i x^{\prime} \cdot \xi+i \tau_{\mu}^{+}\left(\xi ; k_{v}(\xi) \pm i 0\right) x_{n}}{r}\right)^{k} \\
& \quad \times e^{i x \cdot{ }^{\prime} \xi} e^{i \tau_{\mu}^{+}\left(\xi ; k_{v}(\xi) \pm i 0\right) x_{n}} C_{j \mu}^{v}(\eta)+(2 \pi)^{-\frac{n}{2}} e^{i x^{\prime} \xi} \\
& \quad \times \sum_{h=0}^{k} \sum_{l=1}^{m}\left[\left(\left(\begin{array}{l}
k \\
h
\end{array}\right)\left(\frac{i x^{\prime} \cdot \xi}{r}\right)^{h} \frac{}{\Delta(\xi ; \bar{\lambda})} \overline{\operatorname{det}\left(B h_{1}^{+}(\xi ; \bar{\lambda}) \ldots,\right.}\right.\right. \\
& \left.\quad \frac{\partial^{k-h}}{\partial r^{k-h}} B q_{\mu}\left(x_{n}, \xi ; k_{v}(\xi \mp i 0), \ldots, B h_{m}^{+}(\xi ; \bar{\lambda})\right)\right|_{\lambda=k_{v}(\xi) \pm i 0} \\
& \left.\quad \times \gamma_{j l}^{v}(\eta)\right]_{\mu=1, \ldots, 2 m \downarrow}, \quad \eta \in D_{j v}, \quad k=0,1,2, \ldots
\end{aligned}
$$

Here we have used the fact that

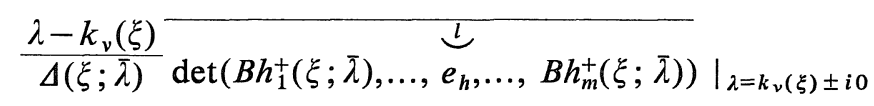

is positively homogeneous of degree 1 , where $e_{h}={ }^{t}(0, \ldots, 1, \ldots, 0)$. We put in [3]

(3.18) $q_{\mu}\left(x_{n}, \xi\right.$; $\left.k_{v}(\xi) \mp i 0\right)$ is equal to the $\mu$-th column vector of

$$
\left(\frac{1}{2 \pi i} \int_{\gamma_{-}} e^{-i x_{n} \tau_{1}}\left(\tau_{1} I-M\left(\xi ; k_{v}(\xi)\right)\right)^{-1} A_{n}^{-1} d \tau_{1}\right)
$$


Thus

(3.19) $\frac{\partial^{h}}{\partial r^{h}} q_{\mu}\left(x_{n}, \xi ; k_{v}(\xi) \mp i 0\right)$ is equal to the $\mu$-th column vector of

$$
\left(\frac{1}{2 \pi i} \int_{\gamma_{-}} \frac{1}{r^{h}}\left[\left(\tau_{1} \frac{d}{d \tau_{1}}\right)^{h} e^{-i x_{n} \tau_{1}}\right]\left(\tau_{1} I-M\left(\xi ; k_{v}(\xi)\right)\right)^{-1} A_{n}^{-1} d \tau_{1}\right)
$$

Hence it follows from (3.19) that

$$
\left|\frac{\partial^{h} q_{\mu}}{\partial r^{h}}\left(x_{n}, \xi ; k_{v}(\xi) \mp i 0\right)\right| \leqq \frac{C_{h}\left(1+|\xi| x_{n}\right)^{h}}{r^{h}}
$$

holds, where the $C_{h}$ are independent of $\left(x_{n}, \xi\right)$. Let us consider the case where $E^{+}(\xi ; 0) \cap \mathscr{B}=\{0\}$ for $|\xi| \neq 0$. Then there exists $\delta(>0)$ such that for all $\xi$ with $|\xi|=1$ and $\xi \in D_{v},\left|k_{v}(\xi)\right|>\delta$. Thus $\tilde{S}_{v}$ are bounded, $1 \leqq v \leqq s$. Therefore, by the same argument as for $I_{j}(r), 1 \leqq j \leqq \rho$, it follows from the estimates of $\Psi_{j+2 v \rho}^{ \pm}(x, \eta)$ in [3] that $I_{j+2 v \rho}(r) \in C^{\infty}[a$, b] for any interval $[a, b], 0<a<b<\infty$, i.e., $I_{j+2 v \rho}(r) \in C^{\infty}(0, \infty)$. Next let us consider the case where $f(x)$ belongs to $C^{\infty}\left(\overline{\mathbf{R}_{+}^{n}}\right)$. Then we have

$$
\begin{gathered}
\left|\mathscr{F}_{x^{\prime}}[p(x) f(x)]\left(\xi, x_{n}\right)\right|=\mid\left(1+|\xi|^{2}\right)^{-l} \mathscr{F}_{x^{\prime}}\left[\left(1-\Delta_{x^{\prime}}\right)^{l}\right. \\
(p(x) f(x))]\left(\xi, x_{n}\right) \mid \leqq C_{l}\left(1+|\xi|^{2}\right)^{-l}
\end{gathered}
$$

for arbitrary non-negative integer $l$ and $p(x) \in C^{\infty}$. Since we have

$$
\begin{aligned}
& \left|C_{j \mu}^{\nu}(\eta)\right| \leqq \frac{C(1+|\xi|)}{1+|\sigma|}, \\
& \mid \gamma_{j l}^{v}(\eta \mid) \leqq \frac{C}{1+|\sigma|},
\end{aligned}
$$

for sufficiently large $R,|\eta|>R$ and $k_{v}(\xi) \in[a, b]$,

$$
\begin{aligned}
& \int_{a \leqq k v(\xi) \leqq b}\left(1+|\xi|^{2}\right)^{-\frac{n}{2}-h-1}\left|\frac{\partial^{h \Psi} \frac{ \pm}{j+2 v \rho}(x, \eta)}{\partial r^{h}}\right|^{2} d \eta<+\infty, \\
& h=0,1,2, \ldots,
\end{aligned}
$$

hold. Thus for $r \in[a, b]$ and $h=0,1,2, \ldots$,

(3.24) $\left|\int_{\mathbf{R}_{+}^{n}}\left(\frac{\partial^{h} \Psi \frac{+}{j+2 v \rho}(x, \eta)}{\partial r^{h}}\right)^{*} f(x) d x\right| \leqq\left(1+|\xi|^{2}\right)^{-l+\frac{h}{2}+\frac{n}{4}+\frac{1}{2}} F_{j+2 v \rho}(\omega)$, 


$$
\left|\int_{\mathbf{R}_{+}^{\eta}}\left(\frac{\partial^{h} \Psi \frac{+}{j+2 v \rho}(x, \eta)}{\partial r^{h}}\right)^{*} f(x) d x\right| \leqq\left(1+|\xi|^{2}\right)^{\frac{h}{2}+\frac{n}{4}+\frac{1}{2}} H_{j+2 v \rho}(\omega),
$$

hold, where

$$
\begin{aligned}
& \int \tilde{S}_{v}\left|\omega \cdot \tilde{n}_{v}(\omega) \| F_{j+2 v \rho}^{h}(\omega)\right|^{2} d \widetilde{S}_{v}<+\infty, \\
& \int_{\tilde{S}_{v}}\left|\omega \cdot \tilde{n}_{v}(\omega) \| H_{j+2 v \rho}^{h}(\omega)\right|^{2} d \tilde{S}_{v}<+\infty .
\end{aligned}
$$

Choosing $l$ more than $\frac{n}{2}+\frac{k}{2}+1$, we have $I_{j+2 v \rho}(r) \in C^{k}(0, \infty)$. Therefore $I_{j+2 v \rho}(r) \in C^{\infty}(0, \infty)$.

Proof of Theorems. Define an orthogonal projection $\widetilde{P}$ on $L^{2}\left(\mathbf{R}_{+}^{n}\right)$ by $\widetilde{P} f(x)=f(x)$ for $|x| \leqq R,=0$ for $|x|>R, f(x) \in L^{2}\left(\mathbf{R}_{+}^{n}\right)$. Let $f \in L^{2}\left(\mathbb{R}_{+}^{n}\right)$ and $f=f_{1}+f_{2}, f_{1}(x)=(P f)(x) \in N(\mathbf{A})^{\perp}, f_{2} \in N(\mathbf{A})$. Then $f$ satisfies $T_{2}$ if and only if $f_{1}=P f$ satisfies $T_{2}$. Suppose that $\operatorname{Supp} f(x)$ is bounded and that at least one of the following conditions holds: a) $E^{+}(\xi ; 0) \cap \mathscr{B}$ $=\{0\}$ for $|\xi| \neq 0$. b) $f(x)$ belongs to $C^{\infty}\left(\overline{\mathbf{R}_{+}^{n}}\right)$. The above arguments and Lemmas 4 and 5 imply that $f$ satisfies $T_{2}$. Thus Theorem 2 follows from Proposition 1. Similarly the first assertion of Theorem 3 follows from Proposition 2. Here although $\tilde{P} R(\lambda) f \rightarrow v^{(-)}(k)$ in $L^{2}\left(\mathbf{R}_{+}^{n}\right)$ as $\lambda \rightarrow k-i 0$ and $\operatorname{Supp}_{x} v^{(-)}(k) \subset\{x ;|x| \leqq R\}$, moving $R$ to $+\infty$, we can define $v^{(-)}(k)$ as follows:

$$
R(\lambda) f \longrightarrow v^{(-)}(k) \text { in } \quad L_{l o c}^{2}\left(\overline{\mathbf{R}_{+}^{n}}\right) \quad \text { as } \quad \lambda \longrightarrow k-i 0 .
$$

Let $\alpha_{R}(x) \in C^{\infty}\left(\mathbf{R}_{+}^{n}\right)$ and $\alpha_{R}(x)=1$ for $|x| \leqq R,=0$ for $|x| \geqq R+1$. Then,

$$
\begin{aligned}
& (\mathbf{A}-k) \alpha_{R}(x) R(\lambda) f=\alpha_{R}(x) f(x)+(\lambda-k) \alpha_{R}(x) R(\lambda) f(x) \\
& \quad+\left(A \alpha_{R}(x)\right)(R(\lambda) f)(x) \longrightarrow \alpha_{R}(x) f(x)+\left(A \alpha_{R}(x)\right) v^{(-)}(x) \\
& \quad \text { in } \quad L^{2}\left(\mathbf{R}_{+}^{n}\right) \text { as } \lambda \longrightarrow k-i 0 .
\end{aligned}
$$

Since $\alpha_{R}(x)(R(\lambda) f)(x) \rightarrow \alpha_{R}(x) v^{(-)}(k)$ in $L^{2}\left(\mathbf{R}_{+}^{n}\right)$ as $\lambda \rightarrow k-i 0$, the closedness of the operator $\mathbf{A}$ implies that $\alpha_{R}(x) v^{(-)}(k) \in D(\mathbf{A})$ and that

$$
(\mathbf{A}-k) \alpha_{R}(x) v^{(-)}(k)=\alpha_{R}(x) f(x)+\left(A \alpha_{R}(x)\right) v^{(-)}(k),
$$

that is, 


$$
\left(A_{l o c}-k\right) v^{(-)}(k)=f(x) .
$$

Moreover suppose that $E^{+}(\xi ; 0) \cap \mathscr{B}=\{0\}$ for $|\xi| \neq 0$. Then $N(\mathbf{A})=\{0\}$ and the coerciveness inequalities for the operator $\mathbf{A}$,

$$
\left\|\frac{\partial u}{\partial x_{j}}\right\| \leqq C(\|\mathbf{A} u\|+\|u\|), j=1, \ldots, n,
$$

hold for $u \in D(\mathbf{A})$. Thus from (3.26) it follows that

$$
R(\lambda) f \longrightarrow v^{(-)}(k) \text { in } H_{1 \mathrm{oc}}^{1}\left(\overline{\mathbf{R}_{+}^{n}}\right) \text { as } \lambda \longrightarrow k-i 0 .
$$

This completes the proof of Theorem 3.

\section{References}

[1] Eidus, D. M., The principle of limit amplitude, Uspehi Mat. Nauk, 24 (1969), 91-156, = Russian Math. Surveys, 24 (1969), 97-167.

[2] Matsumura, M., Comportement asymptotique de solutions de certains problèmes mixtes pour des systèmes hyperboliques symétriques à coefficients constants, Publ. RIMS, Kyoto Univ., 5 (1970), 301-360.

[3] Wakabayashi, S., Eigenfunction expansions for symmetric systems of first order in the half-space $\mathbf{R}_{+}^{n}$, Publ. RIMS, Kyoto Univ., this issue. 